\title{
Detection of abnormal aortic elastic properties in asymptomatic patients with Marfan syndrome by combined transoesophageal echocardiography and acoustic quantification
}

Andreas Franke, Eberhard G Mühler, Heinrich G Klues, Katja Peters, Wolfgang Lepper, Götz von Bernuth, Peter Hanrath

\begin{abstract}
Objective-To evaluate the potential value of transoesophageal echocardiography combined with automated border detection and acoustic quantification for the assessment of elastic properties of the thoracic aorta in patients with Marfan syndrome.
\end{abstract}

Subjects-16 patients with Marfan syndrome and 12 age matched normal controls.

Methods-Transoesophageal echocardiography was performed in all subjects. Minimum and maximum diameters of the descending thoracic aorta were obtained from $M$ mode images and acoustic quantification was used for the on-line evaluation of cross sectional aortic area and peak positive area changes over time. Compliance, distensibility, and stiffness index were calculated using $M$ mode data and non-invasively measured blood pressure and were compared with the indices derived from acoustic quantification.

Results-Aortic dimensions normalised for body surface area were not statistically different between patients and normal controls, but there were significant differences for all elasticity indices except compliance. Marfan patients had a lower distensibility [4.2 (SD 1.8) $\left.v 5.8(2.1) \mathrm{cm}^{2} / \mathrm{dyn}, P<0.05\right]$ and a higher stiffiness index $[9.7(3.0) v 7 \cdot 1$ (1.8), $P<0.05]$. The dynamic indices derived from the acoustic quantification were significantly smaller in Marfan patients [peak positive area change: $5 \cdot 1$ (1.0) $v$ 7.7 (1.7) $\mathrm{cm}^{2} / \mathrm{s} ; P<0.001$; and normalised peak positive area change: $2 \cdot 5(1 \cdot 2)$ $v 4.0(0.8) \mathrm{cm}^{2} / \mathrm{s}$ respectively, $\left.P<0.001\right]$ and were suitable to discriminate between normal and abnormal elastic properties.

Conclusions-In Marfan syndrome elastic properties of the descending aorta are significantly different from normal controls, even in the absence of vessel dilatation. In addition to established static indices, indices derived from acoustic quantification reflect dynamic changes of the cross sectional area for the evaluation of regional vessel mechanics. The on-line assessment of peak positive area change allows differentiation from normal individuals and may be more accurate than standard $M$ mode measurements.
(Heart 1996;75:307-311)

Keywords: Marfan syndrome; transoesophageal echocardiography; acoustic quantification; aortic elastic properties

Prognosis in Marfan syndrome is strongly influenced by cardiovascular complications. The majority of all deaths in this patient group-nearly $75 \%$ - result from aneurysms, dissection or rupture of the thoracic or abdominal aorta, or cardiac failure caused by dilatation of the aortic root and concomitant aortic valve regurgitation. ${ }^{1-3}$ Life expectancy has improved during the last 20 years because of successful cardiovascular surgery in many of these patients. ${ }^{45}$

Vascular pathology in Marfan syndrome is caused by abnormal elastic properties ${ }^{6-8}$ initially not associated with clinical symptoms. These abnormal properties are most likely to be caused by defective synthesis, secretion, and extracellular matrix formation of the fibrillin protein, which is widely distributed in elastic tissues. ${ }^{9}$ The gene coding for fibrillin has been identified on chromosome 15 and is the site of mutations causing Marfan syndrome. ${ }^{910}$

A non-invasive method for the evaluation of elastic properties with reproducible results should be clinically helpful in Marfan patients, especially before morphological changes occur. Transthoracic echocardiographic visualisation of the aorta is limited to the ascending aorta and parts of the aortic arch. ${ }^{11} \mathrm{~A}$ reproducible short axis plane of the ascending aorta cannot usually be achieved. Adequate visualisation of the descending thoracic aorta by the transthoracic approach cannot be obtained. Transoesophageal echocardiography, however, allows optimal visualisation of the thoracic aorta in transverse planes with good image quality ${ }^{11}$ providing detailed information of heart cycle dependent changes of aortic diameter and cross sectional area.

This study was performed to assess the potential value of transoesophageal echocardiography in combination with automated border detection and on-line acoustic quantification in evaluating the elastic properties of the descending thoracic aorta in Marfan patients and normal controls, in comparison with standard measurements of elastic properties (compliance, distensibility, and stiffness 
index) derived from diameters measured by $M$ mode echocardiography.

\section{Methods}

Twenty four consecutive patients with the diagnosis of Marfan syndrome were studied. All fulfilled the diagnostic criteria for Marfan syndrome. ${ }^{12-14}$

Eight of the 24 patients were excluded from the study because there were significant morphological abnormalities of their native descending aorta (two aneurysms, one dissection), because of prosthetic replacement (four patients), or because of severe aortic regurgitation (one patient). The remaining 16 patients had a native descending thoracic aorta without morphological abnormalities and formed the final study group. The demographic data of all the patients included and the normal controls are given in the table. At the time of the study none of the patients was on cardiovascular medication or had mitral or aortic regurgitation. Two patients had previous cardiovascular surgery with replacement of the aortic valve and the ascending aorta. Thirteen of the 16 Marfan patients had a family history of Marfan syndrome.

Demographic and haemodynamic variables of 16 Marfan patients and 12 normal controls. Values are mean (SD) or (range).

\begin{tabular}{lccl}
\hline & Normal subjects & Marfan patients & P value \\
\hline $\mathrm{n}$ & 12 & 16 & \\
Sex & $5 \mathrm{~m}, 7 \mathrm{f}$ & $7 \mathrm{~m}, 9 \mathrm{f}$ & NS \\
Age (years) & $27(9)$ (range 20-57) & $28(13)($ range 12-57) & NS \\
Height (cm) & $174(6)$ & $188(9)$ & $<0 \cdot 001$ \\
Weight (kg) & $58(19)$ & $71(21)$ & NS \\
Body surface area (m²) & $1 \cdot 77(0 \cdot 1)$ & $2 \cdot 00(0 \cdot 17)$ & $<0 \cdot 001$ \\
Functional class & 12 & 14 & \\
$\quad$ No limitation & 0 & 2 & NS \\
NYHA II & $77(8)$ & $80(17)$ & NS \\
Heart rate (beats/min) & $113(14)$ & $120(15)$ & NS \\
Blood pressure (mm Hg) & $64(11)$ & $68(12)$ & NS \\
$\quad$ Systolic & $80(12)$ & $86(12)$ & NS \\
$\quad$ Diastolic & $49(7)$ & $52(10)$ & \\
$\quad$ Mean & & & \\
Pulse pressure & & & \\
\hline
\end{tabular}

NYHA, New York Heart Association

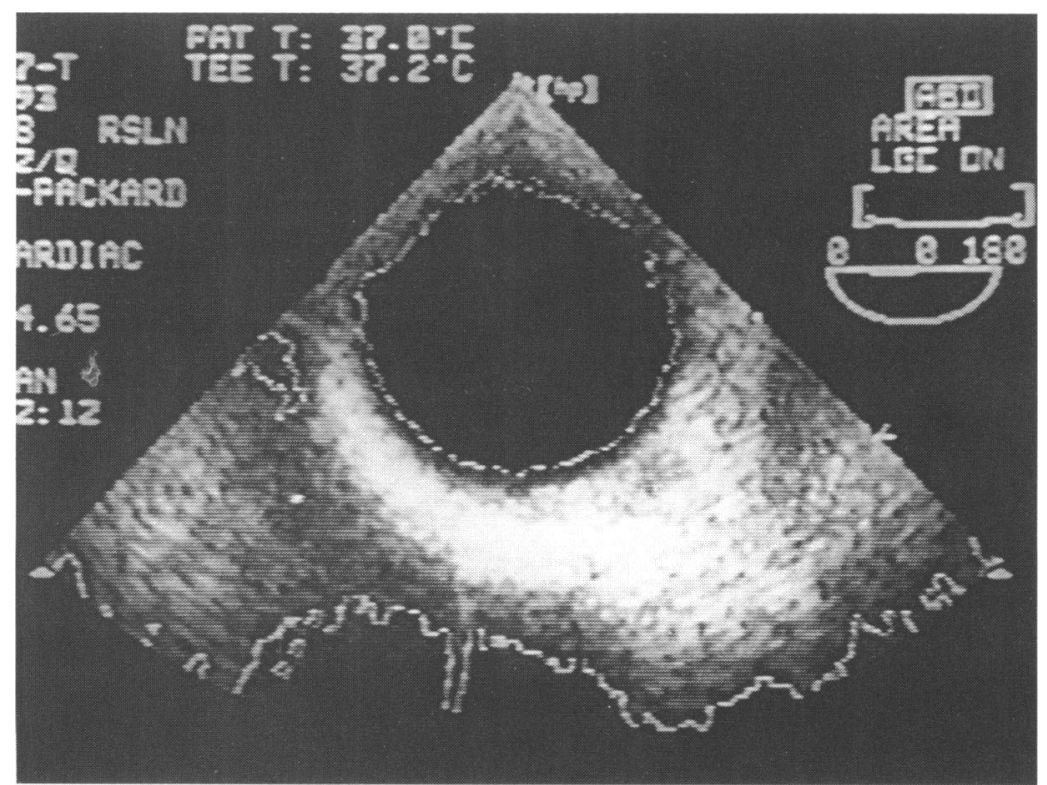

Figure 1 Cross section of the descending aorta with automated border detection. The area within the automatically detected intimal border marked by the thin line is measured online and plotted as a graph.
All patients underwent a complete clinical examination as well as transthoracic and (after given written informed consent) transoesophageal echocardiography (Hewlett Packard Sonos 1500 , with a $2.5 \mathrm{MHz}$ transthoracic and a $5 \mathrm{MHz}$ transoesophageal multiplane transducer). All echocardiographic studies were stored on videotape for further quantitative off-line analysis.

The descending aorta was imaged by transoesophageal echocardiography in an optimised cross sectional strictly circular plane at the level of the left atrium. Great care was taken to visualise the complete circumference of the descending thoracic aorta throughout the whole cardiac cycle.

Minimum and maximum diameters of the descending aorta were determined by conventional $\mathrm{M}$ mode echocardiography. Non-invasive oscillometric blood pressure measurements at the right upper limb were performed every 2 min and the average of five measurements during $M$ mode and acoustic quantification was used for further calculations. The following variables were calculated based on minimum and maximum diameters and blood pressure. ${ }^{15-17}$

Compliance $\left(\mathrm{cm}^{2} / \mathrm{dyn}\right)$

$\mathrm{C}=\frac{\mathrm{A}_{\text {max }}-\mathrm{A}_{\text {min }}}{\mathrm{BP}_{\text {mean }}}$

Distensibility ( $\left.\mathrm{cm}^{2} / \mathrm{dyn}\right)$

$\mathrm{D}=\frac{2 \cdot\left(\mathrm{d}_{\max }-\mathrm{d}_{\min }\right)}{\mathrm{d}_{\min } \cdot\left(\mathrm{BP}_{\text {syst }}-\mathrm{BP}_{\text {diast }}\right)}$

\section{Stiffiness index}

$S I=\frac{\ln \left(B P_{\text {sys }} / B_{\text {diast }}\right)}{\left(d_{\max }-d_{\min }\right) / d_{\min }}$

$A_{\max } ; A_{\min }=$ maximum and minimum cross sectional area.

$\mathrm{d}_{\max } ; \mathrm{d}_{\min }=$ maximum and minimum diameter. $\mathrm{BP}_{\text {syst; }} ; \mathrm{BP}_{\text {diast }} ; \mathrm{BP}_{\text {mean }}=$ systolic, diastolic, and mean blood pressure.

The echocardiographic system used for quantitative integrated backscatter imaging has been described elsewhere. ${ }^{18}$ In brief, backscatter data from each A-line are integrated (over $3.2 \mu \mathrm{s})$ and then used for on-line, real time image reconstruction. Time gain settings were carefully optimised to improve the visualisation of endothelial aortic borders. Lateral gain compensation was not used to avoid artificial underestimation of the aortic cross sectional area. A region of interest was manually traced around the descending thoracic aorta. Integrated software then calculates the identified area along the internal aortic border and displays the results as trace and digital data on the monitor (fig 1). For the present study the minimum and maximum cross sectional area $\left(\mathrm{cm}^{2}\right)$ and peak positive area change $\left(\mathrm{cm}^{2} / \mathrm{s}\right)$ were calculated as an average from five consecutive heart cycles derived from the area and $\mathrm{dA} / \mathrm{dt}$ graphical recording (fig 2).

The normalised peak positive area change (per second) was defined as peak positive area change divided by the minimum cross sectional area:

$\mathrm{dA} / \mathrm{dt}_{\text {norm }}=\frac{\mathrm{dA} / \mathrm{dt}_{\max }}{\mathrm{A}_{\min }}$ 


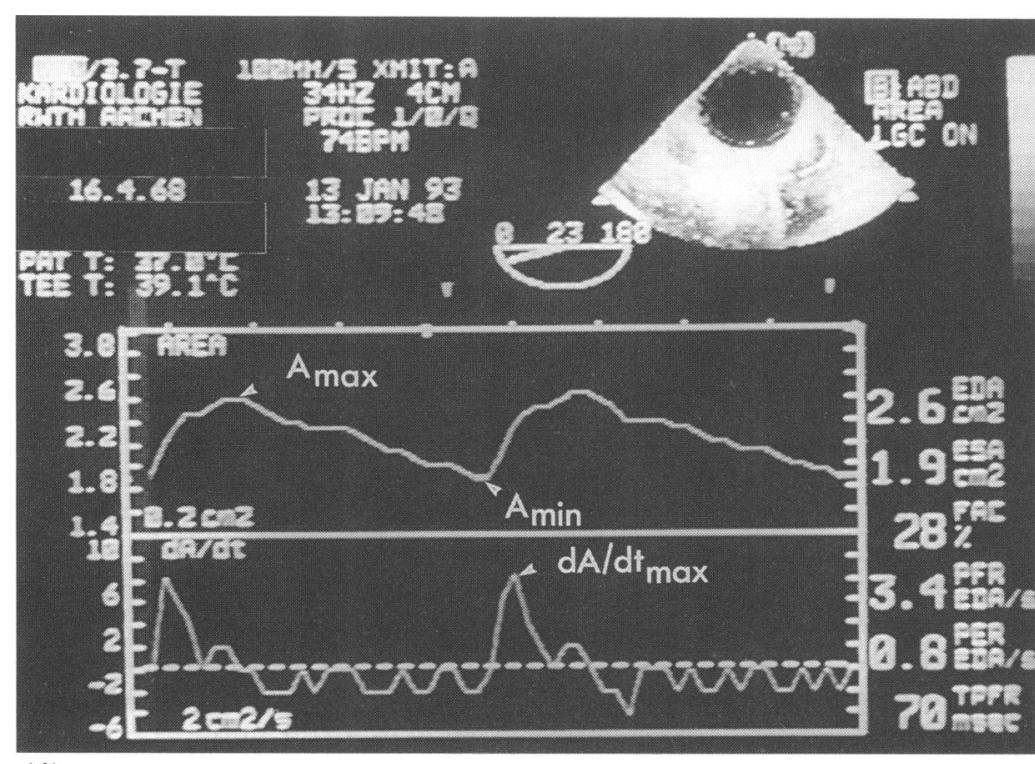

(A)

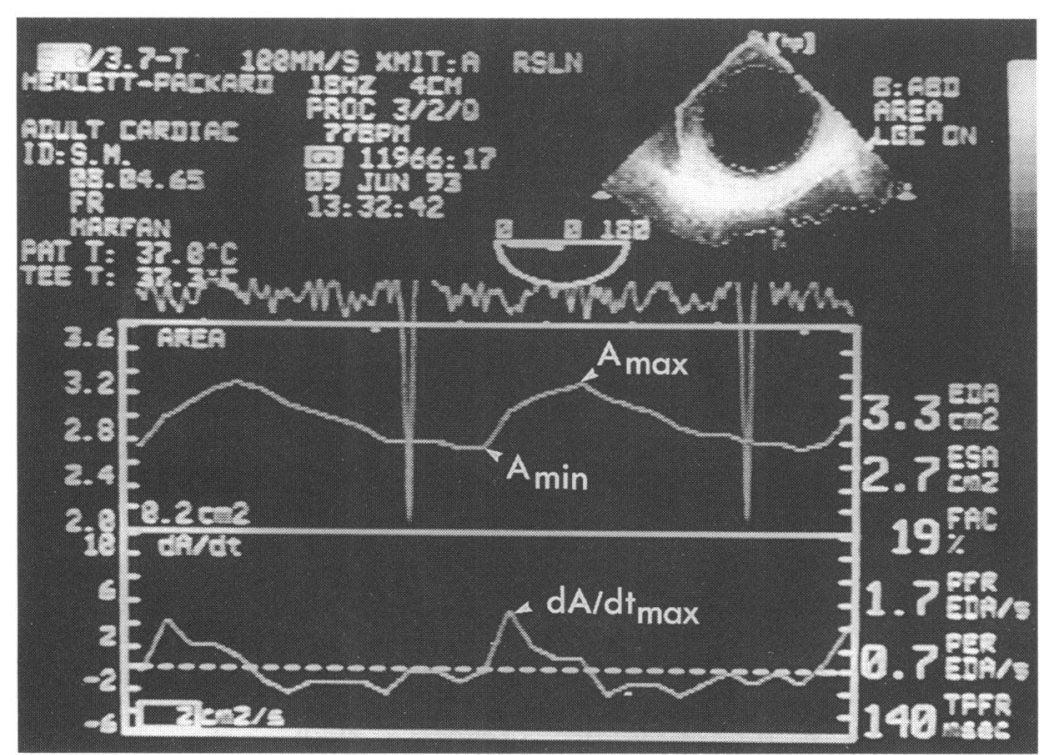

(B)

Figure 2 (A) Descending thoracic aorta of a normal subject with simultaneous acoustic quantification and on-line display of cross sectional area (upper line) and its changes over time (dA/dt, lower line). Peak positive area change (dA/dt $t_{\text {max }}$ ) as a distinct maximum is marked as the cross sectional area increases with arriving pulse wave. Minimum and maximum cross sectional areas are also marked $\left(A_{\text {min }} A_{\text {mon }}\right)$. (B) Imaging of the thoracic aorta in a patient with Marfan syndrome. Note the flattened graph of cross sectional area and the low maxima of the dA/dt graph compared to the normal control.

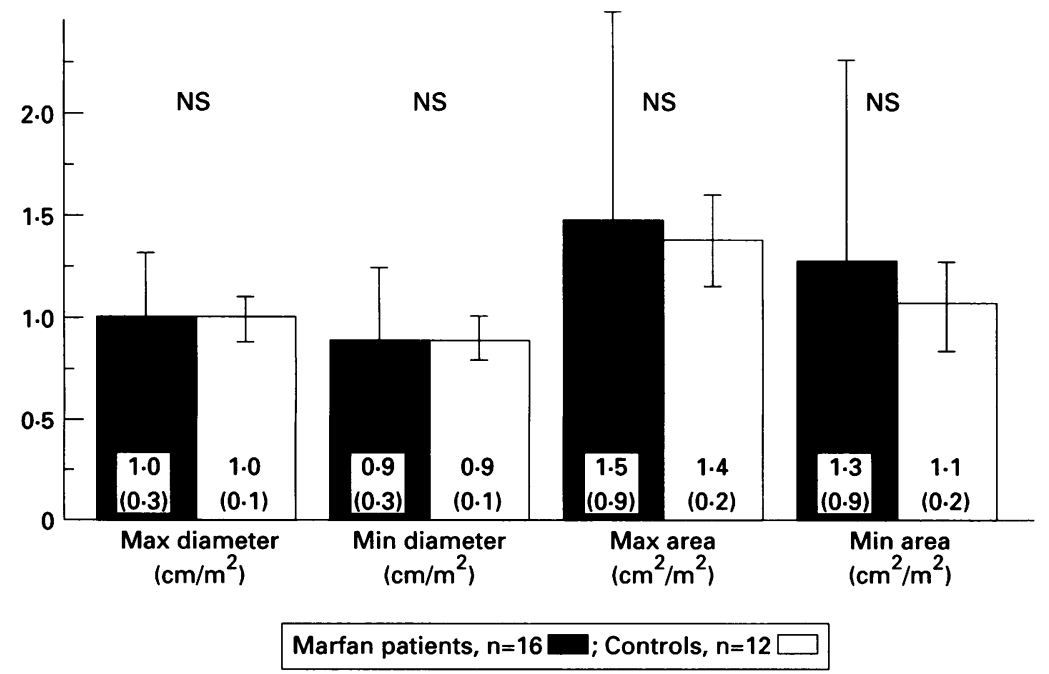

Figure 3 Aortic dimensions of Marfan patients and controls, normalised for body surface area.
For comparison, 12 age matched normal subjects without cardiovascular diseases or connective tissue disorders underwent the same protocol after giving their informed consent.

STATISTICAL ANALYSIS

Data were expressed as mean (SD). An unpaired Student $t$ test and a bivariate linear correlation analysis as well as a $\chi^{2}$ test were used where appropriate to study the relation between different echocardiographic and haemodynamic variables. A P value of less than 0.05 was considered significant.

\section{Results}

DEMOGRAPHIC AND HAEMODYNAMIC VARIABLES There were no significant differences between Marfan patients and controls in age, weight, heart rate, systolic and diastolic blood pressure, or pulse pressure, though there was a slight but not statistically significant tendency for larger pulse pressure in Marfan patients (table). Marfan patients were significantly taller than controls, with corresponding larger body surface area.

\section{MODE BASED INDICES}

There were no significant differences in minimum and maximum aortic diameters and cross sectional areas normalised for body surface (fig 3), even though absolute dimensions were larger in Marfan patients [minimum diameter: 1.9 (SD 0.5) v $1.6(0.2) \mathrm{cm}$; maximum diameter: $2 \cdot 1(0.5) v 1.8(0 \cdot 1) \mathrm{cm}$; minimum cross sectional area: $2.6(1.8) v 2.0$ $(0 \cdot 5) \mathrm{cm}^{2}$; maximum cross sectional area: $3 \cdot 1$ $(1.8) \quad v \quad 2.5 \quad(0.5) \quad \mathrm{cm}^{2} ; \quad \mathrm{P}<0.05$ for all differences].

There was no difference in compliance between both groups (fig 4A), but Marfan

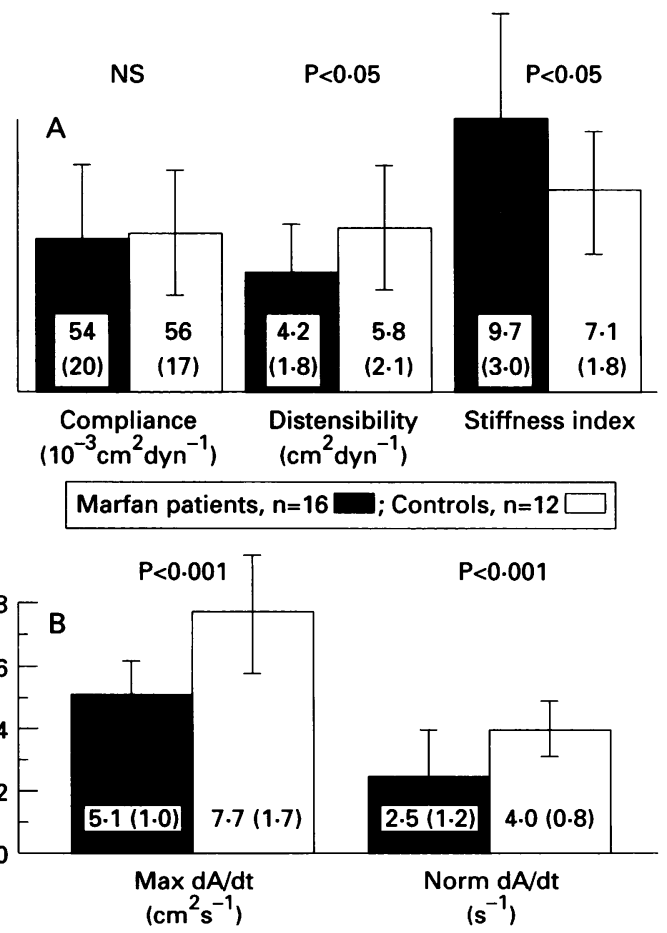

Figure 4 (A) Comparison of both groups for elasticity indices based on $M$ mode measurements. (B) Elasticity indices based on the results of automated border detection. 
patients had a significantly lower distensibility $\left[4 \cdot 2(1 \cdot 8) v 5 \cdot 8(2 \cdot 1) \mathrm{cm}^{2} /\right.$ dyn, $\left.\mathrm{P}<0.05\right]$ and a higher stiffness index than normals $[9 \cdot 7(3 \cdot 0)$ $v 7 \cdot 1(1 \cdot 8), \mathrm{P}<0 \cdot 05]$.

\section{INDICES DERIVED FROM ACOUSTIC}

\section{QUANTIFICATION}

Qualitative individual analysis of the on-line registered cross sectional area graph revealed a flattened curve for Marfan patients compared to normals (fig 2B).

Highly significant differences were obtained between both groups for peak positive area change and normalized peak positive area change (fig 4B). Peak positive area change was lower in Marfan patients than in normals [5.1 (1.0) v $\left.7.7(1.7) \mathrm{cm}^{2} / \mathrm{s}, \mathrm{P}<0.001\right]$. The difference between the mean values of both groups was even higher for normalised peak positive area change $\left[2.5(1.2) v 4.0(0.8) \mathrm{s}^{-1}\right.$, $\mathrm{P}<0.001]$.

\section{LINEAR CORRELATIONS}

There was a good linear correlation between the cross sectional area calculated from $M$ mode derived diameters and the area registered on-line by acoustic quantification (Pearson's $\rho$ correlation coefficient $r=0.82, \quad P<0.001)$. With higher age minimum and maximum diameters as well as cross sectional areas increased (for both: $r=0.60, \mathrm{P}<0.001$ ), distensibility as well as normalised peak positive area change decreased $(r=-0.50, \mathrm{P}<0.01)$ and stiffness index increased (low correlation, $r=0.4$; $\mathrm{P}<0.05)$. There was a low positive correlation between systolic blood pressure and maximum diameters $(r=0.45, \mathrm{P}<0.01)$ and a negative correlation between systolic pressure and distensibility $(r=-0.55$, $P<0.005)$. These correlations apply for the group of Marfan patients as well as to the normal controls.

There was no statistically significant influence of heart rate, diastolic blood pressure, pulse pressure, or previous operation on any of the diameters, areas, or elasticity indices. Even the absolute diameters and cross sectional areas had no statistical influence on the corresponding elasticity indices.

\section{Discussion}

Pathomorphological and functional abnormalities of the thoracic and abdominal aorta are major determinants of the clinical course and prognosis in Marfan syndrome. Therefore, it is important to be able to obtain reliable, reproducible, and non-invasive methods for the early detection of abnormalities in vascular elastic properties. Transoesophageal echocardiography is of proven benefit in the cross sectional visualisation of the thoracic aorta as compared to transthoracic echocardiography. ${ }^{11}$

Until recently measurements of the aortic elastic properties were based either on transthoracic $M$ mode derived calculations, ${ }^{161719}$ on invasive haemodynamic measurements, ${ }^{1620}$ or on magnetic resonance imaging (MRI)..$^{21-23}$

\section{PREVIOUS STUDIES ON VASCULAR ELASTIC} PROPERTIES

Transthoracic and transoesophageal $M$ mode measurements have been used to evaluate the elastic properties of the aortic wall, which has proved to be age dependent in normal individuals. Lower distensibility and a higher stiffness index, calculated from these measurements, have been observed in patients with arterial hypertension, coronary artery disease, ${ }^{172425}$ and Marfan syndrome. ${ }^{6}$

Three recent papers have described the potential value of MRI for evaluating elastic vascular properties and have confirmed the lower distensibility of the thoracic aorta in patients with coronary artery disease ${ }^{21}$ and Marfan syndrome. ${ }^{22} 23$

Intravascular ultrasound techniques have also been used to determine aortic wall stiffness index in animals ${ }^{20}$ and humans. ${ }^{26}$ As for MRI, calculations were based on manual tracing of the minimum and maximum cross sectional area. The invasive character of this method limits its application, especially for serial investigations.

The accuracy of the calculations of elastic property indices with all the methods described above depends on the reliability of vessel diameter or area measurements. In contrast to ventricular measurements, maximum and minimum diameters or areas of the descending aorta are not equal to end systolic and end diastolic diameters or areas; they have to be chosen manually. Obviously it is a problem to select the true minimum and maximum in a circular structure with small area changes. For $\mathbf{M}$ mode measurements, heart cycle dependent motion of the descending thoracic aorta leads to sideshift artefacts causing an underestimation of vessel diameters, because the $M$ mode beam may not be located centrally throughout the whole cardiac cycle. An advantage of the $M$ mode technique, however, is the higher time resolution as compared to acoustic quantification.

\section{PREVIOUS STUDIES USING A COMBINATION}

OF ACOUSTIC QUANTIFICATION AND

TRANSOESOPHAGEAL ECHOCARDIOGRAPHY

Recently introduced automated border detection and acoustic quantification allow the automatic detection and calculation of the maximum and minimum areas of any cardiovascular structure as well as graphical display of the continuous area change during the heart cycle $(\mathrm{dA} / \mathrm{dt})$. First studies using this technique focused on the estimation of left ventricular performance. ${ }^{18}$ Gerber et al used acoustic quantification of the thoracic aorta in combination with transoesophageal echocardiography for the cross sectional analysis of aortic cross sectional area and for the calculation of compliance and distensibility. They found an age dependent decrease of compliance and distensibility as well as slightly increased aortic diameters in healthy subjects. ${ }^{27}$

Our study for the first time uses the combination of transoesophageal echocardiography and acoustic quantification in Marfan patients for the evaluation of elastic properties. The 
results for standard indices (distensibility, stiffness index) are in concordance with those previously reported by Hirata $e t a l^{6}$ and showed a significant difference between Marfan patients and normal controls. We also found a negative correlation between age and distensibility, whereas absolute minimum and maximum diameters or cross sectional areas had no influence on any of the elasticity indices.

The indices that we have now introduced (peak positive area change and normalised peak positive area change)-derived from acoustic quantification and reflecting the dynamic process of area changes-probably discriminate better than standard indices between normal individuals and Marfan patients.

\section{LIMITATIONS}

Measurements of the present study were limited to the descending thoracic aorta. Neighbouring structures such as the left atrium, pulmonary artery, and right ventricle make it too difficult to place the region of interest on the ascending aorta without interference, so that acoustic quantification and on-line measurements of the cross sectional area cannot be performed with the same precision and reliability in the ascending as they can in the descending aorta. There is a problem of limited recognition of intimal borders near to the transducer. This will have more effect on the distance measurements by $M$ mode than on the area measurements using acoustic quantification.

The integrated backscatter technique is limited to a time resolution of about 30 to 35 frames per second. It also requires $3 \cdot 2 \mu$ s per frame for the integration of all A-line data, causing a slight time delay. This delay, however, is not relevant for the present study, because measurements of minimum and maximum diameters and areas were not based on ECG triggering.

FUTURE DIRECTIONS

Combined transoesophageal echocardiography and acoustic quantification for the evaluation of elastic properties of the aorta including indices such as maximum and normalised area change over time might be a good tool for the long term control of Marfan patients. This method seems to be suitable for measuring the influence and postulated benefit of $\beta$ adrenergic blockade ${ }^{28}$ on the elastic properties of vessel walls and might have a prognostic value regarding evolving cardiovascular complications. To prove this, further investigations with a larger group of patients and follow up studies are necessary.

1 Murdoch JL, Walker BA, Halpern BL, Kuzma JW, Mckusick VA. Life expectancy and causes of death in the Marfan's syndrome. N Engl F Med 1972;286:804-8.

2 Roberts WC, Honig HS. The spectrum of cardiovascular disease in the Marfan's syndrome: a clinico-morphologic study of 18 necropsy patients and comparison to 151 previously reported necropsy patients. Am Heart $\mathcal{F} 1982 ; 104$ : 115-35.

3 Marsalese DL, Moodie DS, Vacante M, Lytle BW, Gill CC, Sterba R, et al. Marfan's syndrome: natural history and long-term follow-up of cardiovascular involvement. $\mathcal{F}$ Am Coll Cardiol 1989;14:422-8.

4 Silverman DI, Burton KJ, Gray J, Bosner MS, Kouchoukos NT, Roman MJ, et al. Life expectancy in the Marfan's syndrome. Am $\mathcal{F}$ Cardiol 1995;75:157-160.

5 Gott VL, Cameron DE, Reitz BA, Pyeritz RE. Curren diagnosis and prescription for the Marfan's syndrome: aortic root and valve replacement. $f$ Cardiac Surg 1994;9(suppl 2):177-81.

6 Hirata K, Triposkiadis F, Sparks E, Bowen J, Wooley CF, Boudoulas H. The Marfan syndrome: abnormal aortic elastic properties. F Am Coll Cardiol 1991;18:57-63.

7 Reed CM, Fox ME, Alpert BS. Aortic biomechanical properties in pediatric patients with the Marfan syndrome, and the effects of atenolol. Am $\mathcal{F}$ Cardiol 1993;71:606-8.

8 Savolainen A, Keto P, Hekali P, Nisula L, Kaitila I, Viitasalo $M$, et al. Aortic distensibility in children with the Marfan's syndrome. Am 7 Cardiol 1992;70:691-3.

9 Pereira L, Levran O, Ramirez F, Lynch JR, Sykes B, et al. A molecular approach to the stratification of cardiovascular risk in families with Marfan's syndrome. N Engl $f$ Med 1994;331:148-53

10 Dietz HC, Pyeritz RE, Hall BD, Cadle RG, Hamosha A, Schwartz J. The Marfan's syndrome locus: confirmation of assignment to chromosome 15 and identification of tightly linked markers at 15q15-q21·3. Genomics 1991;9 355-61.

11 Simpson IA, de Belder MA, Treasure T, Camm AJ, Pumphrey $\mathrm{CW}$. Cardiovascular manifestations of Marfan's syndrome: improved evaluation by transoesophageal echocardiography. Br Heart $f$ 1993;69: $104-8$

12 Pyeritz RE, Mckusick VA. The Marfan's syndrome: diagnosis and management. N Engl $\mathcal{F}$ Med 1979;300:772-7.

13 Beighton P, de Paepe A, Dunks D, Finidori G, GeddeDahl T, Goodman R, et al. International nosology of heritable disorders of connective tissue, Berlin 1986. $\mathrm{Am} \mathcal{F}$ Med Genet 1988;29:581-94.

14 Pyeritz RE. Diagnosis of Marfan's syndrome. In: Royce PM, Steinmann B, eds. Connective tissue and its heritable disorders: molecular, genetic and medical aspects. New York: Wiley-Liss, 1993:437-68

15 Hirata K, Triposkiadis F, Sparks E, Bowen J, Boudoula $\mathrm{H}$, Wooley CF. Marfan's syndrome: cardiovascular physical findings and diagnostic correlates. Am Heart $f$ 1992;123:743-57.

16 Stefanadis C, Stratos C, Boudoulas H, Kourouklis C, Toutouzas P. Distensibility of the ascending aorta: comparison of invasive and non-invasive techniques in healthy men and in men with coronary artery disease. Eur Heart $f$ 1990;11:990-6.

17 Stefanadis C, Wooley CF, Bush CA, Kolibash AJ, Boudoulas $\mathrm{H}$. Aortic distensibility abnormalities in coronary artery disease. Am f Cardiol 1987;59:1300-4.

18 Perez JE, Waggoner AD, Barzilai B, Melton HE, Miller JG, Sobel BE. On-line assessment of ventricular function by Sobel BE. On-line assessment of ventricular function by
automatic boundary detection and ultrasonic backscatter imaging. F Am Coll Cardiol 1992;19:313-20.

19 Gamble G, Zorn J, Sanders G, MacMahon S Sharpe N. Estimation of arterial stiffness, compliance and distensibility from $M$-mode ultrasound measurements of the common carotid artery. Stroke 1994;25:11-6.

20 Slørdahl SA, Piene H, Linker DT, Vik A. Segmental aortic wall stiffness from intravascular ultrasound at normal and subnormal aortic pressure in pigs. Acta Physiol Scand 1991;143:227-32.

21 Mohiaddin RH, Underwood SR, Bogren HG, Firmin DN Klipstein RH, Rees RSO, et al. Regional aortic compliance studied by magnetic resonance imaging: the effects of age, training and coronary artery disease. Br Heart 1989;62:90-6.

22 Savolainen A, Keto P, Hekali P, Nisula L, Kaitila I, Viitasal $\mathbf{M}$, et al. Aortic distensibility in children with Marfan's syndrome. Am $₹$ Cardiol 1992;70:691-3.

23 Adams IN, Brooks M, Redpath TW, Smith FW, Dean J, Gray J, et al. Aortic distensibility and stiffness index measured by magnetic resonance imaging in patients with sured by magnetic resonance imaging in patien.

24 Hirai T, Sasayama S, Kawasaki T, Yagi S. Stiffness of systemic arteries in patients with myocardial infarction. temic arteries in patients
Circulation $1989 ; 80: 78-82$.

25 Pasierski TJ, Binkley PF, Pearson AC. Evaluation of aortic distensibility with transoesophageal echocardiography. Am Heart $\mathcal{F}$ 1992;123:1288.

26 Heintz B, Walkenhorst F, Gillessen T, Dörr R, Krebs W vom Dahl $\mathrm{J}$, et al. In vivo characterization of segmenta elastic properties of the aortic tree from intravascula ultrasound. Cardiol Elderly 1994;2:127-32

27 Gerber TC, Foley DA, Greenleaf JF, Tajik J, Seward JB Assessment of age-dependant changes of aortic distens bility with a new acoustic quantification system [abstr]. $\mathcal{F}$ Am Coll Cardiol 1993;21:298A

28 Shores J, Berger KR, Murphy EA, Pyeritz RE. Progression of aortic dilatation and the benefit of long-term betaadrenergic blockade in Marfan's syndrome. N Engl f Med 1994;330:1335-41. 